\title{
Multi-resolution analysis and fractional quantum Hall effect: An equivalence result
}

\author{
F. Bagarello ${ }^{a}$ \\ Dipartimento di Matematica ed Applicazioni, Fac. Ingegneria, Università di Palermo, \\ I-90128 Palermo, Italy
}

(Received 26 January 2001; accepted for publication 10 August 2001)

In this article we prove that any multi-resolution analysis of $\mathcal{L}^{2}(\mathbf{R})$ produces, for some values of the filling factor, a single-electron wave function of the lowest Landau level (LLL) which, together with its (magnetic) translation, gives rise to an orthonormal set in the LLL. We also discuss the inverse construction. Moreover, we extend this procedure to the higher Landau levels and we discuss the analogies and the differences between this procedure and the one previously proposed by J.-P. Antoine and the author. (C) 2001 American Institute of Physics.

[DOI: $10.1063 / 1.1407281]$

\section{INTRODUCTION}

The role of wavelets in various applications of mathematics and to some physical problems like signal analysis is now completely established: the existence of a wide literature on this field is sufficient to give an idea of the amount of people involved in this and related topics. For a clear reading on this subject a standard quotation is Ref. 1 . Reference 2 is an updated book where other interesting aspects of wavelets are discussed. What cannot be found in many textbooks, since is still to be understood, is the relevance of wavelets in quantum mechanics: at this moment, to our knowledge, very few of the applications proposed in this field (Ref. 3-8 among the others).

One of the most useful features of wavelets concerns their localization properties in both configuration and frequency space. This fact is at the basis of a series of papers ${ }^{3-6}$ where different families of orthonormal (o.n.) bases in $\mathcal{L}^{2}(\mathbf{R})$ are used in the search for the ground state of a two-dimensional electron gas (2DEG) in a uniform positive background and subjected to a uniform electro-magnetic field. This is the physical system which produces the well-known fractional quantum Hall effect (FQHE). The key fact behind this approach is the existence of an unitary map between $\mathcal{L}^{2}(\mathbf{R})$ and the lowest Landau level (LLL), that is, the subspace of $\mathcal{L}^{2}\left(\mathbf{R}^{2}\right)$ corresponding to the lowest eigenvalue of the free Hamiltonian of the 2DEG. This implies that any o.n. basis in $\mathcal{L}^{2}(\mathbf{R})$ (not necessarily a wavelet one!) produces an o.n. basis in the LLL; for this reason the role of wavelets does not seem so crucial. We will comment again on this approach in Sec. V.

In this articles we establish a deeper connection between wavelets and FQHE. In particular we will show that any multi-resolution analysis (MRA) of $\mathcal{L}^{2}(\mathbf{R})$ produces automatically a wave function in $\mathcal{L}^{2}(\mathbf{R})$ and, as a second step, a wave function in the LLL which turns out to be o.n. to its own (magnetic) translation. This procedure, which works for an even value of the inverse filling factor, is only possible when we start from a MRA, contrary to what happens in Ref. 3, and can also be inverted: to any o.n. basis in the LLL which is generated by a single wave function via the action of magnetic translations can be associated a MRA.

The article is organized as follows:

In the next section we quickly review some of the main properties of a MRA and of the kq-representation, ${ }^{9}$ which turns out to be a technical tool useful to implement the orthonormality condition.

${ }^{a)}$ Electronic mail: bagarell@unipa.it 
In Sec. III we state the problem of orthonormality of the single electron wave functions in connection with the FQHE.

In Sec. IV we show how, for fillings factors $\nu=1 / 2 L, L \in \mathbf{N}$, a MRA produces in a completely natural way a wave function for the $2 \mathrm{DEG}$ with the desired orthonormality requirement. We also discuss the inverse procedure.

Section $\mathrm{V}$ is devoted to the comparison between this approach and the one proposed in Ref. 3. In particular, the example of the Haar o.n. basis is considered in detail. We also extend our procedure to higher Landau levels.

Section VI contains the conclusions and the plans for the future.

\section{MATHEMATICAL TOOLS}

In order to keep the article self-contained we now quickly review, for the reader's convenience, the main properties of the mathematical tools we will use in the rest of the article.

\section{A. Multi-resolution analysis}

The main result in the theory of MRA is the recipe which allows us to construct an orthonormal basis in $\mathcal{L}^{2}(\mathbf{R})$ starting from a single function $\psi$ and acting on $\psi$ with dilation and translation operators, generating the set

$$
\left\{\psi_{j, k}(x) \equiv 2^{j / 2} \psi\left(2^{j} x-k\right), j, k \in \mathbf{Z}\right\} .
$$

Such a basis has the good properties of wavelets, including space and frequency localization. This is the key to their usefulness in many physical and mathematical applications. Let us now sketch the construction of these o.n. bases of wavelets. The full story may be found, for instance, in Ref. 1.

A multi-resolution analysis of $L^{2}(\mathbf{R})$ is an increasing sequence of closed subspaces

$$
\cdots \subset V_{-2} \subset V_{-1} \subset V_{0} \subset V_{1} \subset V_{2} \subset \cdots,
$$

with $\cup_{j \in Z} V_{j}$ dense in $L^{2}(\mathbf{R})$ and $\cap_{j \in Z} V_{j}=\{0\}$, and such that

(1) $f(x) \in V_{j} \Leftrightarrow f(2 x) \in V_{j+1}$.

(2) There exists a function $\phi \in V_{0}$, called a scaling function, such that $\{\phi(x-k), k \in \mathbf{Z}\}$ is an o.n. basis of $V_{0}$.

Combining (1) and (2), one gets an o.n. basis of $V_{j}$, namely $\left\{\phi_{j, k}(x) \equiv 2^{j / 2} \phi\left(2^{j} x-k\right), k \in \mathbf{Z}\right\}$. The role of $V_{j}$ as an approximation space and in the direct decomposition of $\mathcal{L}(\mathbf{R})$ is discussed in Ref. 1.

Here we only need to know that the theory asserts the existence of a function $\psi$, called the mother of the wavelets, explicitly computable from $\phi$, such that $\left\{\psi_{j, k}(x) \equiv 2^{j / 2} \psi\left(2^{j} x-k\right), j, k\right.$ $\in \mathbf{Z}\}$ constitutes an orthonormal basis of $L^{2}(\mathbf{R})$ : these are the orthonormal wavelets.

The construction of $\psi$ proceeds as follows. First, the inclusion $V_{0} \subset V_{1}$ yields the relation

$$
\phi(x)=\sqrt{2} \sum_{n=-\infty}^{\infty} h_{n} \phi(2 x-n), \quad h_{n}=\left\langle\phi_{1, n} \mid \phi\right\rangle .
$$

Taking Fourier transforms, this gives

$$
\hat{\phi}(\omega)=m_{o}(\omega / 2) \hat{\phi}(\omega / 2),
$$

where

$$
m_{o}(\omega)=\frac{1}{\sqrt{2}} \sum_{-\infty}^{\infty} h_{n} e^{-i n \omega}
$$


is a $2 \pi$-periodic function. Iterating (2.4), one gets the scaling function as the (convergent!) infinite product

$$
\hat{\phi}(\omega)=(2 \pi)^{-1 / 2} \prod_{j=1}^{\infty} m_{o}\left(2^{-j} \omega\right) .
$$

Then one defines the function $\psi \in W_{0} \subset V_{1}$ by the relation

$$
\hat{\psi}(\omega)=e^{i \omega / 2} \overline{m_{o}(\omega / 2+\pi)} \hat{\phi}(\omega / 2),
$$

or, equivalently,

$$
\psi(x)=\sqrt{2} \sum_{n=-\infty}^{\infty}(-1)^{n-1} h_{-n-1} \phi(2 x-n),
$$

and proves that the function $\psi$ indeed generates an o.n. basis with all the required properties.

Actually, this procedure does not produce a unique result. Another possibility, which is the one we will use in the example given later in this work, gives for the mother wavelet the following expansion:

$$
\psi(x)=\sqrt{2} \sum_{n=-\infty}^{\infty}(-1)^{n} h_{-n+1} \phi(2 x-n) .
$$

Various additional conditions may be imposed on the function $\psi$ (hence on the basis wavelets): arbitrary regularity, several vanishing moments (in any case, $\psi$ has always meant zero), fast decrease at infinity, even compact support. For instance, $\psi$ has compact support if only finitely many $h_{n}$ differ from zero.

Simple examples of this construction are the Haar basis, which comes from the scaling function $\phi(x)$ equal to 1 for $0 \leqslant x<1$ and 0 otherwise, the spline functions, ${ }^{1}$ and so on.

What is more interesting for our purposes is the role of the coefficients $\left\{h_{n}\right\}$ defining the two-scale relation (2.3). These are complex quantities which, if $\phi(x)$ is normalized, must satisfy the following relation:

$$
\sum_{n \in \mathbf{Z}}\left|h_{n}\right|^{2}=1
$$

Furthermore, it can be proved using the $2 \pi$-periodicity of the function $m_{o}(\omega)$, together with the orthogonality of the set $\{\phi(x-k)\}$ for $k \in \mathbf{Z}$, that

$$
\left|m_{o}(\omega)\right|^{2}+\left|m_{o}(\omega+\pi)\right|^{2}=1
$$

almost everywhere. ${ }^{1}$ This equation can be written in two equivalent forms where the coefficients $h_{n}$ explicitly appear:

$$
\sum_{n \in \mathbf{Z}} h_{n} \overline{h_{n+2 k}}=\delta_{k, 0}, \quad \forall k \in \mathbf{Z},
$$

or

$$
\sum_{n, k \in \mathbf{Z}} h_{n} \overline{h_{n+2 k}} e^{2, i k \omega}=1, \quad \text { a.e., }
$$

or yet, in a more convenient form, 


$$
\frac{1}{2} \sum_{n, l \in \mathbf{Z}} h_{n} \bar{h}_{l} e^{i(l-n) \omega}\left(1+(-1)^{l+n}\right)=1, \quad \text { a.e. }
$$

We end this rapid excursus on MRA with the following remark: the set of coefficients $\left\{h_{n}\right\}$ can be considered as the main ingredient of a MRA since it generates $m_{o}(\omega), \hat{\phi}(\omega)$ and, finally, the mother wavelet $\psi(x)$.

\section{B. kq-representation}

The relevance of kq-representation in many-body physics has been established since its first appearance. ${ }^{9}$ What was originally a physical tool has become, during the years, also a mathematical interesting object, widely analyzed in the literature, (see Refs. 10 and 11, for instance). We give here only a few definitions and refer to Refs. 9 and 11-13 for further reading and for applications.

The genesis of the kq-representation consists in the well-known possibility of a simultaneous diagonalization of any two commuting operators. In Ref. 12 it is shown that the following distributions,

$$
\psi_{k q}(x)=\sqrt{\frac{2 \pi}{a}} \sum_{n \in \mathbf{Z}} e^{i k n a} \delta(x-q-n a), \quad k \in\left[0, a\left[, \quad q \in\left[0, \frac{2 \pi}{a}[,\right.\right.\right.
$$

are (generalized) eigenstates of both $T(a)=e^{i p a}$ and $\tau(2 \pi / a)=e^{i x 2 \pi / a}$. Here $a$ is a positive real number which plays the role of a lattice spacing.

How it is discussed in Ref. 12, these $\psi_{k q}(x)$ are Bloch-like functions corresponding to infinitely localized Wannier functions. They also satisfy orthogonality and closure properties. This implies that, roughly speaking, they can be used to define a new representation of the wave functions by means of the integral transform $Z: \mathcal{L}^{2}(\mathbf{R}) \rightarrow \mathcal{L}^{2}(\square)$, where $\square=[0, a[\times[0,2 \pi / a[$, defined as follows:

$$
h(k, q):=(Z H)(k ; q):=\int_{\mathbf{R}} d \omega \overline{\psi_{k q}(\omega)} H(\omega),
$$

for all functions $H(\omega) \in \mathcal{L}^{2}(\mathbf{R})$. The result is a function $h(k, q) \in \mathcal{L}^{2}(\square)$.

To be more rigorous, $Z$ should be defined first on the functions of $\mathcal{C}_{0}^{\infty}(\mathbf{R})$ and then extended to $\mathcal{L}^{2}(\mathbf{R})$ using its continuity. ${ }^{10}$ In this way it is possible to give a rigorous meaning to formula (2.16).

From now on we will work in the following hypothesis:

$$
a^{2}=2 \pi,
$$

which, also in view of the next section, will correspond to fixing the spacing of the lattice underlying the 2DEG.

Replacing $\psi_{k q}(x)$ with its explicit expression, formula (2.16) produces

$$
h(k, q)=(Z H)(k, q):=\frac{1}{\sqrt{a}} \sum_{n \in \mathbf{Z}} e^{-i k n a} H(q+n a),
$$

which can be inverted and gives the $x$-representation $H(\omega) \in \mathcal{L}^{2}(\mathbf{R})$ of a function $h(k, q)$ $\in \mathcal{L}^{2}(\square)$ as follows:

$$
H(\omega)=\left(Z^{-1} h\right)(\omega)=\int_{\square} d k d q \psi_{k q}(\omega) h(k, q) .
$$

Due to (2.15), this equation gives 


$$
H(x+n a)=\frac{1}{\sqrt{a}} \int_{0}^{a} d k e^{i k n a} h(k, x), \quad \forall x \in[0, a[, \quad \forall n \in \mathbf{Z} .
$$

In all the literature concerning kq-representation, the role of the boundary conditions is widely discussed, also in connection with the continuity properties of the functions. For instance, in Ref. 14, a function $h(k, q) \in \mathcal{L}^{2}(\square)$ is said to be continuous if it is the restriction to the kq-cell of a function continuous in the extended kq-plane $(k, q \in \mathbf{R})$, and if it satisfies the following boundary conditions:

$$
\begin{gathered}
h(k+a, q)=h(k, q), \\
h(k, q+a)=e^{i k a} h(k, q),
\end{gathered}
$$

which are typical of any function in kq-representation and which will always be assumed here.

\section{STATING THE PROBLEM}

In this section we will discuss a many-body model of the FQHE looking, in particular, for the single-electron wave function which generates the ground state of the physical system in the way described next. This system is simply a two-dimensional electron gas, 2DEG (that is a gas of electrons constrained in a two-dimensional layer), in a positive uniform background and subjected to a uniform magnetic field along $z$ and an electric field along $y$.

The Hamiltonian of the system can be written as

$$
H^{(N)}=H_{0}^{(N)}+\lambda\left(H_{c}^{(N)}+H_{B}^{(N)}\right)
$$

where $H_{0}^{(N)}$ is the sum of $N$ contributions:

$$
H_{0}^{(N)}=\sum_{i=1}^{N} H_{0}(i)
$$

Here $H_{0}(i)$ describes the minimal coupling of the electrons with the fields:

$$
H_{0}=\frac{1}{2}(\underline{p}+\underline{A}(r))^{2}=\frac{1}{2}\left(p_{x}-\frac{y}{2}\right)^{2}+\frac{1}{2}\left(p_{y}+\frac{x}{2}\right)^{2} \text {. }
$$

Notice that we are adopting here the symmetric gauge $\underset{A}{A}=1 / 2(-y, x, 0)$ and the same unit as in Ref. 15. $H_{c}^{(N)}$ is the canonical Coulomb interaction between charged particles:

$$
H_{c}^{(N)}=\frac{1}{2} \sum_{i \neq j}^{N} \frac{1}{\left|\underline{r}_{i}-\underline{r}_{j}\right|}
$$

and $H_{B}^{(N)}$ is the interaction of the charges with the background, whose explicit form can be found in Ref. 15.

In the following we will consider, as it is usually done in the literature, $\lambda\left(H_{c}^{(N)}+H_{B}^{(N)}\right)$ as a perturbation of the free Hamiltonian $H_{0}^{(N)}$, and we will look for eigenstates of $H_{0}^{(N)}$ in the form of Slater determinant built up single electron wave functions. This approach is known to give good results for low electron (or hole) densities. ${ }^{15}$ The easiest way to attack this problem consists in introducing the new variables

$$
P^{\prime}=p_{x}-y / 2, \quad Q^{\prime}=p_{y}+x / 2 .
$$

In terms of $P^{\prime}$ and $Q^{\prime}$ the single electron Hamiltonian, $H_{0}$, can be written as 


$$
H_{0}=\frac{1}{2}\left(Q^{\prime 2}+P^{\prime 2}\right) \text {. }
$$

The transformation (3.5) can be seen as a part of a canonical map from $\left(x, y, p_{x}, p_{y}\right)$ into $\left(Q, P, Q^{\prime}, P^{\prime}\right)$ where

$$
P=p_{y}-x / 2, \quad Q=p_{x}+y / 2 .
$$

These operators satisfy the following commutation relations:

$$
[Q, P]=\left[Q^{\prime}, P^{\prime}\right]=i, \quad\left[Q, P^{\prime}\right]=\left[Q^{\prime}, P\right]=\left[Q, Q^{\prime}\right]=\left[P, P^{\prime}\right]=0 .
$$

It is shown in Refs. 16 and 17 that a wave function in the $(x, y)$-space is related to its $P P^{\prime}$-expression by the formula

$$
\Psi(x, y)=\frac{e^{i x y / 2}}{2 \pi} \int_{-\infty}^{\infty} \int_{-\infty}^{\infty} e^{i\left(x P^{\prime}+y P+P P^{\prime}\right)} \Psi\left(P, P^{\prime}\right) d P d P^{\prime} .
$$

The usefulness of the $P P^{\prime}$-representation stems from the expression (3.6) of $H_{0}$. Indeed, in this representation, the single electron Schrödinger equation admits eigenvectors $\Psi\left(P, P^{\prime}\right)$ of $H_{0}$ of the form $\Psi\left(P, P^{\prime}\right)=f\left(P^{\prime}\right) h(P)$. Thus the ground state of (3.6) must have the form $f_{0}\left(P^{\prime}\right) h(P)$, where

$$
f_{0}\left(P^{\prime}\right)=\pi^{-1 / 4} e^{-P^{\prime 2} / 2},
$$

and the function $h(P)$ is arbitrary, which manifests the degeneracy of the LLL. With $f_{0}$ as above, formula (3.9) becomes

$$
\psi(x, y)=\frac{e^{i x y / 2}}{\sqrt{2} \pi^{3 / 4}} \int_{-\infty}^{\infty} e^{i y P} e^{-(x+P)^{2} / 2} h(P) d P .
$$

It is worthwhile to stress that at this stage the Coulomb interaction has not yet been considered (and it will not in this article), but the common belief is that the explicit form of $h(P)$ should be fixed by this interaction.

Now the problem arises of how to construct the ground state of the free $N$-electron Hamiltonian $H_{0}^{(N)}$. We use a suggestion coming from the classical counterpart of this quantum problem. It is very well known that the ground state for a classical 2DEG is a (triangular) Wigner crystal: the classical electrons are sharply localized on the sites of a lattice whose lattice spacing is fixed by the electron density. What we expect, and what was proven in Ref. 15, is that, at least for certain regions of the filling factor, the quantum ground state should not be very different from this classical picture. Here we only sketch the procedure which is analyzed in more detail Refs. 15 and 3.

We start introducing the so-called magnetic translation operators $T\left(\vec{a}_{i}\right)$ defined by

$$
T\left(\vec{a}_{i}\right) \equiv \exp \left(i \vec{\Pi}_{c} \cdot \vec{a}_{i}\right), \quad i=1,2,
$$

where $\vec{\Pi}_{c} \equiv(Q, P)$ and $\vec{a}_{i}$ are the lattice basis vectors $\left[\vec{a}_{1}=a(1,0), \vec{a}_{2}=(a / 2)(1, \sqrt{3})\right.$ for a triangular lattice].

From now on, for simplicity we will work in a square lattice with unit cell of area $2 \pi$ :

$$
\vec{a}_{1}=a(1,0), \quad \vec{a}_{2}=a(0,1), \quad a^{2}=2 \pi .
$$

This choice is quite useful to keep the notation simple: moreover, its generalization to lattices of arbitrary shape is only a technical step.

The above rationality condition on the area has the following useful consequence: 


$$
\left[T\left(\vec{a}_{1}\right), T\left(\vec{a}_{2}\right)\right]=0 .
$$

This is not the only commutativity condition satisfied by the magnetic translations. Due to the commutation relations (3.8), we also find

$$
\left[T\left(\vec{a}_{1}\right), H_{0}\right]=\left[T\left(\vec{a}_{2}\right), H_{0}\right]=0 .
$$

With the choice (3.13) of the lattice's basis the magnetic translations take a simple form

$$
T_{1}:=T\left(\vec{a}_{1}\right)=e^{i a Q}, \quad T_{2}:=T\left(\vec{a}_{2}\right)=e^{i a P},
$$

and they act on a generic function $f(x, y) \in \mathcal{L}^{2}\left(\mathbf{R}^{2}\right)$ as follows

$$
f_{m, n}(x, y):=T_{1}^{m} T_{2}^{n} f(x, y)=(-1)^{m n} e^{i(a / 2)(m y-n x)} f(x+m a, y+n a) .
$$

We see from this formula that, if for instance $f(x, y)$ is localized around the origin, then $f_{m, n}(x, y)$ is localized around the lattice site $a(-m,-n)$.

Now we have all the ingredients to construct the ground state of $H_{0}^{(N)}$ mimiking the classical procedure. We simply start from the single electron ground state of $H_{0}$ given in $(3.11), \psi(x, y)$. Then we construct a set of copies $\psi_{m, n}(x, y)$ of $\psi$ as in (3.17), with $m, n \in \mathbf{Z}$. All these functions still belong to the lowest Landau level for any choice of the function $h(P)$ due to (3.15). $N$ of these wave functions $\psi_{m, n}(x, y)$ are finally used to construct a Slater determinant for the finite system:

$$
\psi^{(N)}\left(\underline{r}_{1}, r_{2}, \ldots, r_{N}\right)=\frac{1}{\sqrt{N !}}\left|\begin{array}{cccc}
\psi_{m_{1}, n_{1}}\left(r_{1}\right) & \psi_{m_{1}, n_{1}}\left(r_{2}\right) & \ldots & \psi_{m_{1}, n_{1}}\left(r_{N}\right) \\
\psi_{m_{2}, n_{2}}\left(r_{1}\right) & \psi_{m_{2}, n_{2}}\left(r_{2}\right) & \ldots & \psi_{m_{2}, n_{2}}\left(r_{N}\right) \\
\cdot & \cdot & \ldots & . \\
\cdot & . & \ldots & . \\
\cdot & . & \ldots & . \\
\psi_{m_{N}, n_{N}{ }_{-}\left(r_{1}\right)} & \psi_{m_{N}, n_{N}}\left(r_{2}\right) & \ldots & \psi_{m_{N}, n_{N}}\left(r_{N}\right)
\end{array}\right|
$$

It is known ${ }^{15}$ that in order to get $\left\langle\psi^{(N)}, \psi^{(N)}\right\rangle=1$ we need to have

$$
\left\langle\psi_{m_{i}, n_{i}} \psi_{m_{j}, n_{j}}\right\rangle=\delta_{m_{i}, m_{j}} \delta_{n_{i} n_{j}}
$$

In fact, if these translated functions were not o.n., then we would get $\left\|\psi^{(N)}\right\|=1+O(N)$, which is obviously divergent for $N$ diverging. It is clear, therefore, that if we want to perform easily the thermodynamical limit, orthonormality between differently localized single electron wave functions must be required!

In the rest of this section we will discuss how the requirement (3.19) can be handled and, in particular, we will show that the use of kq-representation is quite a useful tool since it produces a very simple constraint. Some of the results we are now going to describe in this section are also due to G. Morchio and F. Strocchi, ${ }^{18}$ while the original idea of using kq-representation in connection with an orthonormality constraint is already contained in Ref. 13 in the proof of completeness of lattice states proposed by the authors.

Let $\psi(x, y)$ be as in (3.11) and $\psi_{m, n}(x, y)=T_{1}^{m} T_{2}^{n} \psi(x, y)=(-1)^{m n} e^{i(a / 2)(m y-n x)} \psi(x+m a, y$ $+n a)$. After few computations and using the rationality condition $a^{2}=2 \pi$ we obtain

$$
\psi_{m, n}(x, y)=\frac{e^{i(x y / 2)+i a m y}}{\sqrt{2} \pi^{3 / 4}} \int_{-\infty}^{\infty} d P e^{i(y+n a) P-(x+m a+P)^{2} / 2} h(P)
$$


We are interested now in finding some conditions on $h(P)$ such that condition (3.19), or its equivalent form

$$
S_{m, n}:=\left\langle\psi_{0,0}, \psi_{m, n}\right\rangle=\delta_{m, 0} \delta_{n, 0},
$$

is satisfied. With the previous definitions we find

$$
S_{m, n}=\int_{-\infty}^{\infty} d p e^{i n a p} \overline{h(p+m a)} h(p),
$$

which restates the problem of the orthonormality of the wave functions in terms of the $P P^{\prime}$-representation. In particular we see that, for $m=n=0$, this equation implies that $\psi$ in normalized in $\mathcal{L}^{2}\left(\mathbf{R}^{2}\right)$ if and only if $h(P)$ is normalized in $\mathcal{L}^{2}(\mathbf{R})$. This reflects the unitarity of the transformation (3.9), which, more in general, implies that any o.n. set in $\mathcal{L}^{2}(\mathbf{R})$ is mapped in an o.n. set in $\mathcal{L}^{2}\left(\mathbf{R}^{2}\right)$.

In order to use now kq-representation it is convenient to split the integral over $\mathbf{R}$ into an infinite sum of integrals restricted to $[r a,(r+1) a[, r \in \mathbf{Z}$, use the kq-representation, and, then, write everything in terms of a single integral over the unit cell $\square$. We have, using (2.20) and the well-known equality

$$
\begin{gathered}
\sum_{l \in \mathbf{Z}} e^{i x l(2 \pi / c)}=c \sum_{l \in \mathbf{Z}} \delta(x-c l), \\
S_{m, n}=\sum_{r \in \mathbf{Z}} \int_{r a}^{(r+1) a} d p e^{i n a p} \overline{h(p+m a)} h(p) \\
=\sum_{r \in \mathbf{Z}} e^{i n r a^{2}} \int_{0}^{a} d p e^{i n a p} \overline{h(p+(r+m) a)} h(p+r a) \\
=\sum_{r \in \mathbf{Z}} \frac{1}{a} \int_{0}^{a} d q \int_{0}^{a} d k \int_{0}^{a} d k^{\prime} e^{i r\left(k-k^{\prime}\right) a} e^{i n a q-i k^{\prime} m a} h(k, q) \overline{h\left(k^{\prime}, q\right)},
\end{gathered}
$$

so that

$$
S_{m, n}=\int_{\square} d k d q e^{i n a q-i k m a}|h(k, q)|^{2}
$$

Due to the completeness of the set $\left\{e^{i n a q-i k m a}, n, m \in \mathbf{Z}\right\}$ in the unit cell $\square$, we conclude that $S_{m, n}=\delta_{m, 0} \delta_{n, 0}$ if and only if $h(k, q)$ is a phase, so that $|h(k, q)|$ is independent of $k$ and $q$. This result can be considered as a slight generalization of the procedure discussed in Ref. 13 to the FQHE for filling factor $\nu=1$.

It is easy to generalize this result to a filling $\nu=1 / 2$. The idea is the following:

A filling factor $\nu=1$ corresponds to all the sites of our square lattice (of spacing $a=\sqrt{2 \pi}$ ) occupied. A $\nu=1 / 2$ 2DEG can be seen, on the other hand, as if the same lattice was only partially occupied: one lattice site is free and the other is occupied. If we require the orthonormality of the related set of single electron wave functions, it is enough to ask for $S_{m, 2 n}=\delta_{m, 0} \delta_{n, 0}$. This is equivalent also to choose a different lattice, with a unit cell twice that before and basis vectors $a(1,0)$ and $2 a(0,1)$. Of course, we would as well have chosen another lattice with basis vectors $a(0,1)$ and $2 a(1,0)$, or also any other lattice with unit cell of area $4 \pi$. We use the first choice just to fix ideas. Equation (3.24) gives

$$
S_{m, 2 n}=\int_{\square} d k d q e^{i 2 n a q-i k m a}|h(k, q)|^{2}=\delta_{m, 0} \delta_{n, 0},
$$


which can be rewritten as

$$
\frac{1}{2} \int_{\square} d k d q e^{i n a q-i k m a}\left(\left|h\left(k, \frac{q}{2}\right)\right|^{2}+\left|h\left(k, \frac{q+a}{2}\right)\right|^{2}\right) .
$$

This implies, again using the completeness of the functions $e^{i n a q-i k m a}, n, m \in \mathbf{Z}$, in $\square$, that

$$
J_{2}(k, q):=\left|h\left(k, \frac{q}{2}\right)\right|^{2}+\left|h\left(k, \frac{q+a}{2}\right)\right|^{2}=\frac{1}{\pi}, \quad \text { almost everywhere for } k, q \in \square .
$$

The generalization to $\nu=1 / M$ is straightforward: we simply require the orthonormality of the wave functions located at a distance of $M$ sites:

$$
S_{m, M n}=\int_{\square} d k d q e^{i M n a q-i k m a}|h(k, q)|^{2}=\delta_{m, 0} \delta_{n, 0}
$$

and, proceding as above, we deduce that $h(k, q)$ must satisfy the equality

$$
J_{M}(k, q):=\left|h\left(k, \frac{q}{M}\right)\right|^{2}+\left|h\left(k, \frac{q+a}{M}\right)\right|^{2}+\cdots+\left|h\left(k, \frac{q+(M-1) a}{M}\right)\right|^{2}=\frac{M}{2 \pi},
$$

almost everywhere for $k, q \in \square$.

The extension to a filling $\nu=L / M$, with $L$ and $M$ relatively prime, can be performed by imposing that condition $S_{m, n}=\delta_{m, 0} \delta_{n, 0}$ holds only for those $(m, n)$ corresponding to a square lattice in which only $L$ among $M$ lattice sites are occupied. We will not consider this extension in this article.

\section{WHAT WE GET FROM MRA}

In this section we will describe how two subjects which are so different, at a first sight, as the MRA and the orthonormality condition for a 2DEG discussed previously, are indeed very close.

Let us consider a given MRA of $\mathcal{L}^{2}(\mathbf{R})$. We have seen in Sec. II that to this MRA is associated a certain set of square-summable complex numbers $\left\{h_{n}\right\}_{n \in Z}$ satisfying, for instance, condition (2.12). This set produces a $2 \pi$-periodic function $m_{o}(\omega)$ and, through this, the scaling function $\hat{\phi}(\omega)$ and the mother wavelet.

Now we use the sequence $\left\{h_{n}\right\}_{n \in \mathbf{Z}}$ to define the following function, which strongly reminds us of $m_{o}(\omega)$ :

$$
T_{2}(\omega)=\left\{\begin{array}{l}
\frac{1}{\sqrt{a}} \sum_{l \in \mathbf{Z}} h_{l} e^{-i l \omega a}, \quad \omega \in[0, a[ \\
0, \quad \text { otherwise. }
\end{array}\right.
$$

It is clear that $T_{2}(\omega)$ is square integrable and is not periodic. In particular, due to the normalization condition (2.10), we have $\left\|T_{2}\right\|_{2}^{2}=\int_{\mathbf{R}}\left|T_{2}(\omega)\right|^{2} d \omega=1$. Therefore the kq-transform of this function, $t_{2}(k, q)=\left(Z T_{2}\right)(k, q)$, is well defined in $\mathcal{L}^{2}(\square)$.

In particular, using (2.18) we find

$$
t_{2}(k, q)=\frac{1}{\sqrt{a}} \sum_{n \in \mathbf{Z}} e^{-i k n a} T_{2}(q+n a)
$$

The boundary conditions (2.21) are obviously satisfied: $t_{2}(k+a, q)=t_{2}(k, q)$ and $t_{2}(k, q+a)$ $=e^{i k a} t_{2}(k, q)$. It is easy to check that $t_{2}(k, q)$ satisfies also the orthonormality conditions (3.27). In fact, since we are interested to the value of $t_{2}(k, q)$ only in $\square$, and since $T_{2}(\omega)$ is different from zero only for $\omega \in[0, a[$, we conclude that, for $(k, q) \in \square$, 


$$
J_{2}(k, q)=\frac{1}{a}\left(\left|T\left(\frac{q}{2}\right)\right|^{2}+\left|T\left(\frac{q+a}{2}\right)\right|^{2}\right)=\frac{1}{a^{2}} \sum_{l, s} h_{l} \overline{h_{s}} e^{i(s-l) q a / 2}\left(1+(-1)^{l+s}\right),
$$

which is equal to $1 / \pi$ a.e. in $k, q \in \square$, due to (2.14). This implies that $t_{2}(k, q)$ gives rise to a family of functions $\psi_{m, n}(x, y)$ in the LLL mutually orthonormal and corresponding to $\nu=\frac{1}{2}$. We will find the explicit form of these $\psi_{m, n}(x, y)$ in the next section, where we will also compare these results with the ones obtained in Ref. 3.

The above-mentioned procedure can be easily extended to fillings $\nu=1 / 2 L$. The extension to odd denumerator is not so straightforward and will be given elsewhere.

The starting point is again the set $\left\{h_{n}\right\}_{n \in Z}$, producing a MRA of $\mathcal{L}^{2}(\mathbf{R})$, satisfying condition (2.12). Now we define

$$
T_{2 L}(\omega)=\left\{\begin{array}{l}
\frac{1}{\sqrt{a}} \sum_{l \in \mathbf{Z}} h_{l} e^{-i l \omega L a}, \quad \omega \in[0, a[ \\
0, \quad \text { otherwise }
\end{array}\right.
$$

Again, this is a square-integrable function satisfying $\left\|T_{2 L}\right\|^{2}=1$. Defining $t_{2 L}(k, q)=\left(Z T_{2 L}\right)$ $\times(k, q)$ we have, for $k, q \in \square, t_{2 L}(k, q)=(1 / \sqrt{a}) T_{2 L}(q)=(1 / a) \Sigma_{l \in Z} h_{l} e^{-i l q L a}$. We also stress that $t_{2 L}(k, q)$ satisfies the correct boundary conditions. With these definitions, using the rationality conditions $a^{2}=2 \pi$ and collecting contributions of the form $\left|t_{2 L}(k, q / 2 L)\right|^{2}, \quad \mid t_{2 L}(k,(q$ $+2 a) / 2 L)\left.\right|^{2}, \ldots$, and the "odd ones," $\left|t_{2 L}(k,(q+a) / 2 L)\right|^{2},\left|t_{2 L}(k,(q+3 a) / 2 L)\right|^{2}, \ldots$, we obtain

$$
\begin{aligned}
J_{2 L}(k, q) & :=\left|t_{2 L}\left(k, \frac{q}{2 L}\right)\right|^{2}+\left|t_{2 L}\left(k, \frac{q+a}{2 L}\right)\right|^{2}+\cdots+\left|t_{2 L}\left(k, \frac{q+(2 L-1) a}{2 L}\right)\right|^{2} \\
& =L\left(\left|t_{2 L}\left(k, \frac{q}{2 L}\right)\right|^{2}+\left|t_{2 L}\left(k, \frac{q+a}{2 L}\right)\right|^{2}\right) \\
& =\frac{L}{a^{2}} \sum_{l, s} h_{l} \bar{h}_{s} e^{i(s-l) q a / 2}\left(1+(-1)^{l+s}\right),
\end{aligned}
$$

which is again independent of $k$ and $q$ since it is equal to $L / \pi$ a.e. in $\square$, due to condition (2.14). Finally, Eq. (3.28) is a consequence of the equality $\nu^{-1}=M=2 L$. We conclude that $t_{2 L}(k, q)$ produces, in the configuration space, a set of mutually orthonormal wave functions spanning the LLL for $\nu=1 / 2 L$.

This result, which is in a certain sense rather unexpected because it relates two distant fields as MRA and FQHE, is only half of the surprise. In fact, in the rest of this section, we will also show that this relation works in the opposite direction. More in detail, we will show how to construct, starting from a function $h(k, q)$ which produces an o.n. set of translated functions in the LLL, a set of coefficients $\left\{h_{n}\right\}$ satisfying condition (2.14), and, therefore, generating a MRA.

The recipe is rather simple and requires only few lines: let us suppose to have a function $h(k, q)$ belonging to $\mathcal{L}^{2}(\square)$ satisfying the boundary conditions $h(k+a, q)=h(k, q)$ and $h(k, q$ $+a)=e^{i k a} h(k, q)$ and such that

$$
|h(k, q / 2)|^{2}+|h(k,(q+a) / 2)|^{2}=\frac{1}{\pi} \quad \text { a.e. in } \square .
$$

This means that in the configuration space the related set $\left\{\psi_{m, n}(x, y)\right\}$ is an o.n. set. Let us now define

$$
h_{n}(k)=\int_{0}^{a} d q e^{i n a q} h(k, q), \quad k \in[0, a[
$$


Even if $h_{n}(k)$ is, in general, a function of $k$, it is straightforward to check that if we take $h(k, q)$ coinciding with $t_{2}(k, q)$ in (4.2), then $h_{n}(k)=h_{n}$ for all $n \in \mathbf{Z}$. This means that the dependence on $k$ may disappear in some relevant situation. It is not so surprising, therefore, to check that $\Sigma_{n \in \mathbf{Z}} h_{n}(k) \overline{h_{n+2 l}(k)}$ does not depend on $k$ for any choice of $h(k, q)$, if the equality (4.5) is satisfied. In fact, using equality (3.23) and condition (4.5), we find

$$
\begin{aligned}
\sum_{n \in \mathbf{Z}} h_{n}(k) \overline{h_{n+2 l}(k)} & =a \int_{0}^{a} d q|h(k, q)|^{2} e^{-2 i l a q} \\
& =\frac{a}{2} \int_{0}^{a} d q e^{-i l a q}\left(|h(k, q / 2)|^{2}+|h(k,(q+a) / 2)|^{2}\right) \\
& =\frac{a}{2 \pi} \int_{0}^{a} d q e^{-i l a q}=\delta_{l, 0} .
\end{aligned}
$$

This result shows that any o.n. basis in the LLL for a filling factor $\nu=1 / 2$ produces a set of coefficients satisfying the summation rule (2.12) and, therefore, the basic condition giving rise to a MRA of $\mathcal{L}^{2}(\mathbf{R})$ (which, in general, will depend on an external parameter $k \in[0, a[$ ). The extension to a filling $\nu=1 / 2 L, L \in \mathbf{N}$, is straightforward.

\section{EXTENSION TO HIGHER LANDAU LEVELS AND FURTHER REMARKS}

In the first part of this section we analyze the relation between the approach we have discussed here with the one originally proposed in Ref. 3 and further developed in Refs. 4 and 5. In those papers we used wavelet analysis in connection with the FQHE as we have done here. In Ref. 5, in particular, we discussed a toy model suggesting the relevance of single electron wave functions arising from wavelet theory in the construction of a Slater-like ground state for a 2DEG. This construction was carried out in detail for the FQHE in Refs. 3 and 4 using the canonical transformation (3.11) and the $P P^{\prime}$-representation to generate an o.n. basis of functions in the LLL starting from an o.n. set of wavelets in $\mathcal{L}^{2}(\mathbf{R})$. This procedure is only apparently close to the one proposed in this article. The first difference is related to the possibility of extending the approach in Ref. 3 to any o.n. basis of $\mathcal{L}^{2}(\mathbf{R})$, a possibility which does not exist here since the procedure proposed in this article only works for an o.n. basis generated by a MRA. The second difference concerns the nature of the operators acting on the mother function which generates the o.n. set in the LLL: in Refs. 3 and 4 these operators are dilation and translation operators. Here, on the other hand, we use the magnetic translations defined in (3.12).

Since, however, these two procedures have something in common, we expect that the resulting wave functions should not be very different. And, in fact, this is the outcome of this section, where we will explore the details of the easiest example: the Haar wavelet. For this choice the set $\left\{h_{n}\right\}_{n \in \mathbf{Z}}$ reduces to $h_{0}=h_{1}=1 / \sqrt{2}$, and all the other coefficients are zero. We have shown in Ref. 3 that this choice produces a function in the LLL localized around the origin which looks like

$$
H_{00}(x, y)=\frac{e^{-i x y / 2} e^{-y^{2} / 2}}{2 \pi^{1 / 4}}\left\{2 \phi\left(\frac{x-i y+1 / 2}{\sqrt{2}}\right)-\phi\left(\frac{x-i y}{\sqrt{2}}\right)-\phi\left(\frac{x-i y+1}{\sqrt{2}}\right)\right\},
$$

where $\phi(z):=(2 / \sqrt{\pi}) \int_{0}^{z} e^{-t^{2}} d t$ is the error function. ${ }^{19}$ The whole set $H_{m n}(x, y)$ is discussed in Ref. 3, where its asymptotic behavior is also discussed in connection with the localization of the electrons. Here we only state the result which will be compared with the one resulting by the approach proposed here. We have

$$
H_{00}(x, y) \simeq \frac{e^{i x y / 2} e^{-x^{2} / 2}}{2 \pi^{1 / 4}} \sqrt{\frac{2}{\pi}}\left(\frac{1}{x-i y}+\frac{e^{-1 / 2-x+i y}}{x-i y+1}-2 \frac{e^{-1 / 8-(x-i y) / 2}}{x-i y+1 / 2}\right),
$$

which displays the Gaussian localization of the wave function in the variable $x$ and shows the rather poor localization in $y$. 
Let us now proceed in a different way. For a filling $\nu=1 / 2$ and a generic MRA, the function $T_{2}$ which produces an o.n. set of translates in the LLL is given in (4.1). Using the transformation rule (3.11) we obtain

$$
T_{2}(x, y)=\frac{e^{i x y / 2}}{\sqrt{2} \pi^{3 / 4}} \int_{-\infty}^{\infty} e^{i y Q-(x+Q)^{2} / 2} T_{2}(Q)=\frac{\sqrt{a} e^{i x y / 2}}{2 \pi^{3 / 4}} \sum_{l \in \mathbf{Z}} h_{l} \int_{0}^{a} e^{i Q(y-l a)-(x+Q)^{2} / 2},
$$

which, for the above choice of coefficients corresponding to the Haar wavelet, gives

$$
T_{2}(x, y)=\frac{\sqrt{a} e^{i x y / 2}}{2^{3 / 2} \pi^{5 / 4}} \int_{0}^{a} e^{i Q y-(x+Q)^{2} / 2}\left(1+e^{-i Q a}\right) d Q .
$$

Here $T_{2}$ can be written in terms of error function $\phi(z)$ as follows:

$$
\begin{aligned}
T_{2}(x, y)= & \frac{\sqrt{a} e^{-i x y / 2-y^{2} / 2}}{4 \pi^{3 / 4}}\left(\phi\left(\frac{x+a-i y}{\sqrt{2}}\right)+\phi\left(\frac{x+a-i(y-a)}{\sqrt{2}}\right)-\phi\left(\frac{x-i y}{\sqrt{2}}\right)\right. \\
& \left.-\phi\left(\frac{x-i(y-a)}{\sqrt{2}}\right)\right),
\end{aligned}
$$

whose asymptotic behavior can be found with the help of Ref. 19:

$$
T_{2}(x, y) \simeq \frac{\sqrt{a} e^{+i x y / 2-x^{2} / 2}}{2^{3 / 2} \pi^{5 / 4}}\left(\frac{1}{x-i y}+\frac{e^{\pi-i a(x-i y)}}{x-i(y-a)}-\frac{e^{-\pi-a(x-i y)}}{x+(a-i y)}-\frac{e^{-a(x-i y)(1+i)}}{x+a-i(y-a)}\right) .
$$

This formula shows that, even if the two procedures produce different results, the asymptotic behaviors, that is, the localization features of the electrons, coincide for $H_{00}$ and $T_{2}$. This result can be considered as a consequence of the Balian-Low theorem applied to the present situation (see Refs. 1 and 6) and of the Battle theorem for our previous proposal (see Refs. 3, 20, and 6). Both these theorems give severe constraints on the localization properties of a wave function when orthonormality requirements of a different kind are imposed. We refer to Ref. 6 for a rather complete review of the localization problem in a generic Landau level.

The function $T_{2}$ can be used to construct a Slater determinant for the $N$-electron system as sketched above: we start considering its (magnetic) translated as in (3.17),

$$
\begin{aligned}
\left(T_{2}\right)_{m, n}(x, y)= & \frac{\sqrt{a} e^{-i x y / 2-i a n x-(y+n a)^{2} / 2}}{4 \pi^{3 / 4}}\left\{\phi\left(\frac{x+(m+1) a-i(y+n a)}{\sqrt{2}}\right)-\phi\left(\frac{x+m a-i(y+n a)}{\sqrt{2}}\right)\right. \\
& \left.+\phi\left(\frac{x+(m+1) a-i(y+(n-1) a)}{\sqrt{2}}\right)-\phi\left(\frac{x+m a-i(y+(n-1) a)}{\sqrt{2}}\right)\right\} .
\end{aligned}
$$

These are the functions used to build up the antisymmetric wave function

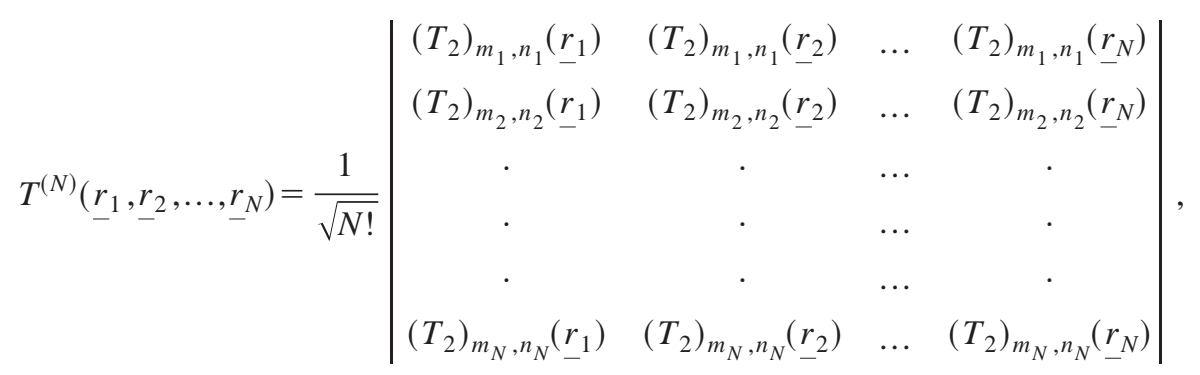

where $\left(m_{i}, n_{i}\right)$ are those indexes compatible with an electron density $\nu=1 / 2$. 
It is evident that our procedure produces many possible $N$-electron wave functions in the LLL, one for each different MRA of $\mathcal{L}^{2}(\mathbf{R})$. Among all these possibilities, the one physically relevant is that choice which minimizes the Coulomb energy. Of course, before comparing these results with those obtained using the Laughlin wave function, we first need to generalize our procedure to a triangular lattice. The details of this extension will be considered in a future work.

In the last part of this section we extend the orthonormality constraint (3.21) to levels higher than the lowest.

We begin this analysis with a general remark, which already suggests the final result: orthonormality is required on a set of functions obtained by a single wave function via the action of the magnetic translations $T_{i}$. On the other hand, the passage from a Landau level to the other is obtained with the action of the raising and lowering operators $A^{\prime \dagger}$ and $A^{\prime}$ defined by

$$
A^{\prime}=\frac{Q^{\prime}+i P^{\prime}}{\sqrt{2}}
$$

where $Q^{\prime}$ and $P^{\prime}$ are given in (3.5). We have already remarked that the translations $T_{i}$ commute with $Q^{\prime}$ and $P^{\prime}$, and with $A^{\prime}$ and $A^{\prime \dagger}$ as a consequence, so that it is reasonable to expect that the orthonormality constraint does not change very much moving from the lowest to some higher Landau level. This is exactly what happens, as we will now show explicitly for the first excited level.

All the wave functions of the first Landau level, ILL, are given by formula (3.9) with $\Psi\left(P, P^{\prime}\right)=f_{1}\left(P^{\prime}\right) h(P)$. Here $f_{1}\left(P^{\prime}\right)=\left(\sqrt{2} / \pi^{1 / 4}\right) P^{\prime} e^{-P^{\prime 2} / 2}$ is the first excited function of the harmonic oscillator. Performing the integration in $P^{\prime}$ we obtain

$$
\psi(x, y)=\frac{i e^{-i x y / 2}}{\pi^{3 / 4}} \int_{-\infty}^{\infty} e^{i y P} e^{-P^{2} / 2} P h(P-x) d P .
$$

Acting on $\psi(x, y)$ with $T_{i}$ as in (3.17) and defining $S_{m, n}$ as in (3.21) we obtain

$$
\begin{aligned}
S_{m, n} & =\frac{1}{\pi^{3 / 2}} \int d^{2} r \int_{-\infty}^{\infty} d p \int_{-\infty}^{\infty} d p^{\prime} e^{-i a n x-i y p+i(y+n a) p^{\prime}-\left(p^{2}+p^{\prime 2}\right) / 2} p p^{\prime} \overline{h(p-x)} h\left(p^{\prime}-x-m a\right) \\
& =\frac{2}{\sqrt{\pi}} \int_{-\infty}^{\infty} d x \int_{-\infty}^{\infty} d q e^{i n a q} \overline{h(q)} h(q-m a)(q+x)^{2} e^{-(q+x)^{2}} \\
& =\int_{-\infty}^{\infty} d p e^{i n a p} \overline{h(p+m a)} h(p),
\end{aligned}
$$

which coincides with the result obtained for the LLL. This means that, when passing to the kq-representation, the wave function originating the o.n. set in the ILL is exactly the same function originating the o.n. set in the LLL. Needless to say, this does not imply that in the configuration space the two different o.n. sets coincide, because they are generated by different $\psi\left(P, P^{\prime}\right)$, belonging to different Landau levels.

Even if the above-mentioned result has been obtained only for the ILL, it gives a strong indication that the orthonormality condition in terms of $h(P)$ takes exactly the same form for all the Landau levels. This also follows form our original remark on the commutativity among $T_{i}$ and $A^{\prime \dagger}$.

\section{OUTCOME}

In this article we have proven a deep connection between a MRA of $\mathcal{L}^{2}(\mathbf{R})$ and the FQHE. In particular we have shown how a single electron wave function, which, together with its magnetic translates, produces an o.n. set in the LLL, can be constructed starting from a MRA. This proce- 
dure works for $\nu=1 / 2 L, L \in \mathbf{N}$. We have also shown that this procedure can be essentially inverted since to any o.n. basis of translated functions of the LLL (corresponding to $\nu=1 / 2 L$ ) corresponds a set of coefficients satisfying the main condition of a MRA of $\mathcal{L}^{2}(\mathbf{R})$. Moreover, we have compared this approach with a similar one, Ref. 3 , which is close for the final result but is very different for the philosophy. We have finally extended this procedure to other Landau levels.

What is still to be done is a computation of the energy of the 2DEG for such a basis, in order to see if this procedure can give some hints about the ground state for the FQHE. We also plan to extend this procedure to filling $\nu$ of the form $\nu=1 /(2 L+1)$ and, more generally, $\nu=L / L^{\prime}$, with $L$ and $L^{\prime}$ relatively prime natural numbers.

\section{ACKNOWLEDGMENT}

This work has been financially supported by M.U.R.S.T.

${ }^{1}$ I. Daubechies, Ten Lectures on Wavelets (Society for Industrial and Applied Mathematics, Philadelphia, 1992).

${ }^{2}$ H. L. Resnikoff and R. O. Wells, Jr., Wavelet Analysis (Springer-Verlag, New York, 1998).

${ }^{3}$ F. Bagarello and J. P. Antoine, "Wavelet-like orthonormal basis of the lowest Landau level," J. Phys. A 27, 2471-2481 (1994).

${ }^{4}$ F. Bagarello, "More wavelet-like orthonormal bases for the lowest Landau level: Some considerations," J. Phys. A 27, 5583-5597 (1994).

${ }^{5}$ F. Bagarello, "Applications of wavelets to quantum mechanics: A pedagogical example," J. Phys. A 29, 565-576 (1996).

${ }^{6}$ F. Bagarello and J. P. Antoine, "Localization properties and wavelet-like orthonormal bases for the lowest Landau level," in Advances in Gabor Analysis, edited by H. G. Feichtinger and T. Strohmer (Birkhäuser, Boston, to appear).

${ }^{7}$ K. Cho, T. A. Arias, and J. D. Joannopoulos, "Wavelets in electronic structure calculations," Phys. Rev. Lett. 71, 1808 (1993).

${ }^{8}$ L. De Windt, P. Fisher, M. Defranceschi, J. Delhalle, and J. G. Fripiat, “A combined analytical and numerical strategy to solve the atomic Hartree-Fock equations in momentum space," J. Comput. Phys. 111, 266 (1994).

${ }^{9}$ J. Zak, "Dynamics of electrons in solids in external fields," Phys. Rev. 168, 686 (1968).

${ }^{10}$ I. Daubechies, A. Grossmann, and Y. Meyer, "Rainless nonorthogonal expansions," J. Math. Phys. 27, 1271 (1986).

${ }^{11}$ A. J. E. M. Janssen, "Bargmann transform, Zak transform, and coherent states," J. Math. Phys. 23, 720 (1982).

${ }^{12}$ J. Zak, "The kq-representation in the dynamics of electrons in solids," in Solid State Physics, edited by H. Ehrenreich, F. Seitz, and D. Turnbull (Academic, New York, 1972), Vol. 27.

${ }^{13}$ H. Bacry, A. Grossmann, and J. Zak, "Proof of completeness of lattice states in the kq representation," Phys. Rev. B 12, 1118 (1975).

${ }^{14}$ M. Boon and J. Zak, “Amplitutes on von Neumann lattices," J. Math. Phys. 22, 1090 (1981).

${ }^{15}$ F. Bagarello, G. Morchio, and F. Strocchi, "Quantum corrections to the Wigner crystal. A Hartree-Fock expansion," Phys. Rev. B 48, 5306 (1993).

${ }^{16}$ I. Dana and J. Zak, "Adams representation and localization in a magnetic field," Phys. Rev. B 28, 811 (1983).

${ }^{17}$ M. Moshinsky and C. Quesne, "Linear canonical transformations and their unitary representations," J. Math. Phys. 12, 1772 (1971).

${ }^{18}$ F. Bagarello, G. Morchio, and F. Strocchi, internal report.

${ }^{19}$ I. S. Gradshteyn and I. M. Ryzhik, Table of Integrals, Series and Products (Academic, New York, 1980).

${ }^{20}$ G. Battle, "Phase space localization theorem for ondelettes," J. Math. Phys. 30, 2195 (1989). 